\title{
Artificial Intelligence Technology in Intelligent Health Informatics
}

\author{
Abdel-Badeeh M. Salem ${ }^{(\varpi)}$ \\ Head of Artificial Intelligence and Knowledge Engineering Research Labs \\ Faculty of Computer and Information Sciences, Ain Shams University, \\ Abbassia Cairo, Egypt \\ absalem@cis.asu.edu.eg, abmsalem@yahoo.com \\ http://staff. asu.edu.eg/Badeeh-Salem \\ http://aiasulab. OoOwebhostapp.com/
}

\begin{abstract}
Artificial Intelligence (AI) is devoted to creating computer software and hardware that imitates the human mind. The primary goal of AI technology is to make computers smarter by creating software that will allow a computer to mimic some of the functions of the human brain in selected applications. Applications of AI technology include; general problem solving, expert systems, natural language processing, computer vision, robotics, and education. All of these applications employ knowledge base and inferencing techniques to solve problems or help make decisions in specific domains.
\end{abstract}

In the last years various AI paradigms and computational intelligence (CI) techniques have been proposed by the researchers in order to develop efficient and smart systems in the areas of health informatics and health monitoring systems.AI and CI offers robust, intelligent algorithms and smart methods that can help to solve problems in a variety of health and life sciences areas.

This talk discusses the potential role of the AI and CI approaches, techniques, and theories which are used in developing the intelligent health informatics and health monitoring systems. The talk presents the following techniques: (a) case-based reasoning; (b) intelligent data mining; (c) rough sets; (d) genetic algorithms; and (e) ontological engineering. Strengths and weaknesses of these approaches were enunciated. Also, the talk presents the challenges as well as the current research directions in the areas of health informatics. Examples of the research performed by the author and his associates for developing knowledge-based systems for cancer, heart, brain tumor, and thrombosis diseases are discussed. 